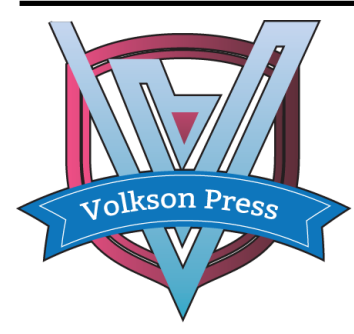

Contents List available at VOLKSON PRESS

New Materials and Intelligent Manufacturing (NMIM) DOI : http://doi.org/10.26480/icnmim.01.2018.143.145

Journal Homepage: https://topicsonchemeng.org.my/

ISBN: 978-1-948012-12-6

\title{
DESIGN OF TARGET VALUE FILTER TYPE TWO-DEGREE OF FREEDOM PID CONTROLLER
}

\author{
Sun Mingge ${ }^{1}$, Zhao Junming ${ }^{1}$, Ren Juan ${ }^{2}$, Li Jiayi ${ }^{*}$ \\ ${ }^{1}$ College of Information \& Control Engineering, Jilin Institute of Chemical Technology, Chengde Street, Jilin, China \\ ${ }^{2}$ Jilin Koito Dongguang Automobile Lamp Co., Ltd., Xiangshan Road, Jilin, China \\ *Corresponding Author Email: washy59@sina.com
}

This is an open access article distributed under the Creative Commons Attribution License, which permits unrestricted use, distribution, and reproduction in any medium, provided the original work is properly cited

\section{ARTICLE DETAILS}

Article History:

Received 26 June 2018

Accepted 2 July 2018

Available online 1 August 2018

\section{ABSTRACT}

Taking the electric oven as temperature control object, this paper proposes a target value filter type two-degree freedom PID control scheme based on two-degree freedom PID control theory. This method improves the inadequacy of conventional PID control in temperature control system, makes the system have the abilities to resist disturbance and track objectives. The model of the oven is obtained by diagraming the tested step response. Then establish the SIMULINK model of the control system, simulate and analyze the robustness. Simulation results show the effectiveness and feasibility of the method.

\section{KEYWORDS}

Target value filter, Two-degree freedom, Robustness, Temperature control

\section{INTRODUCTION}

Due to the significance of temperature as a vital physical quantity in industry, agriculture production, science experimental research and daily lives, temperature control has a wide-spread application in various fields of industry engineering including steel production as well as daily lives [1]. To guarantee the top quality, high yield, low consumption and safety in production, it is necessary and meaningful to accurately measure and effectively control temperature. PID control takes more than 80 percent in common temperature control schemes for the simplicity and easy implementation [2]. It can also meet the performance request in most cases. However, the conventional PID control fails to achieve good performance of control system in both set-point tracking and disturbance rejection. Two-Degree Freedom PID (TDF-PID) Control, as the result of advanced and upgraded PID control, overcomes the shortage of the conventional one-degree of freedom PID controller by combining it with compensation factors [3]. TDF-PID Control takes into account both the requests for performance in set-point tracking and disturbance rejection, achieves the control performance improvement as well as easy adjustment (i.e. both the best performance can be obtained by adjusting three IPD parameters as same as the original one-degree IPD in quantity) [4]. With only the insertion of target value filter, TDF-PID Control is also simple in construction and easy to implement.

In this paper, we research electric oven as a typical temperature control system, and choose the Changdi brand CK-10 attemperating and timing electric oven produced by Westa Electric Appliances Co., Ltd. Of Foshan. It is hard to establish an accurate model of electric oven for its strongnonlinearity, time-varying and long-time-lag features. Since conventional PID controller can hardly reach the high control accuracy, we design target value filter type two-degree of freedom PID controller to control the temperature of electric oven. Our technical requirements include short adjust time, zero-shoot and steady-state error within $\pm 1^{\circ} \mathrm{C}$.

\section{MODELING}

Electric oven has heat capacity and the inner temperature increases gradually when the heater is powered on. When the temperature of the heater strip rises by degrees, the temperature of the oven box goes up by thermal transmission and thermal radiation of the walls. Considering the box has a certain lagging capacity and other components can be considered as proportional cycles, the mathematical model of the temperature control object can be generally described as a first-order inertia added with a lagging link. So we obtain the general form

$$
\mathrm{G}(s)=\frac{k e^{-\tau s}}{T s+1}
$$

where $k$ is static gain, $\tau$ is lag time constant, $T$ is inertia time constant.

The three characteristic parameters above can usually be determined by step response. Add a step signal to the system at a certain power to obtain the system step response curve (Figure 1), and then calculate the characteristic parameters by the Cohn-Coon formula

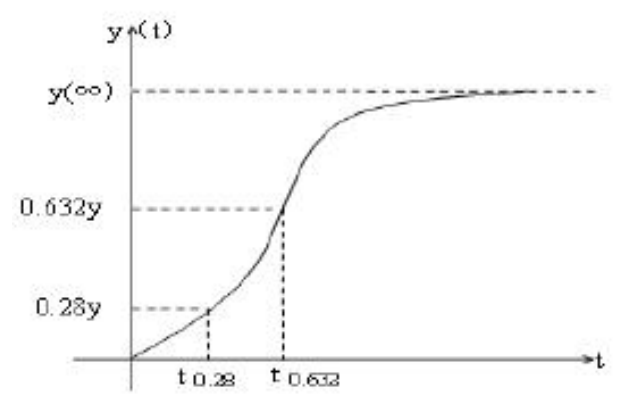

Figure 1: Step Response Curve of Electric Oven

$$
\begin{gathered}
k=\frac{\Delta o u t}{\Delta i n} \\
\tau=1.5\left(t_{0.632}-t_{0.28}\right) \\
T=1.5\left(t_{0.28}-\frac{1}{3} t_{0.632}\right)
\end{gathered}
$$

where $t_{0.28}$ and $t_{0.632}$ denote the rising time mapping $0.28 y$ and $0.632 y$ in the step response curve. 
The experimental object is the Changdi brand CK-10 attemperating and timing electric oven produced by Westa Electric Appliances Co., Ltd. Of Foshan. After determining the system parameters by diagraming method, we choose $\tau=1.5, k=3, T=5$ [5].

\section{CONTROLLER DESIGN}

The controller is designed as a target value filter type two-degree of freedom PID controller. The structure is depicted in Figure 2.

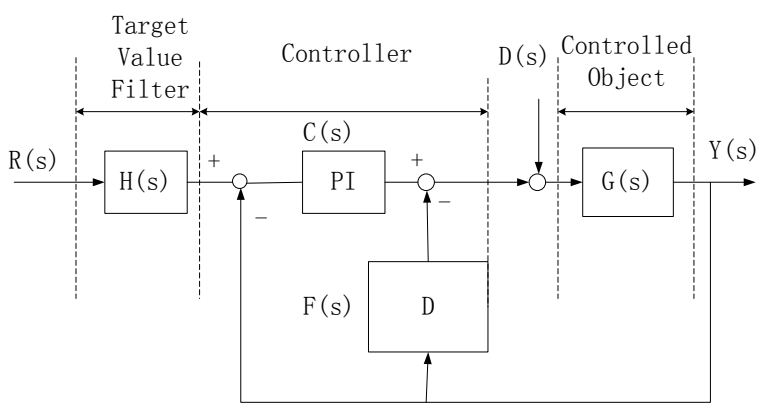

Figure 2: Target Value Filter Type TDF-PID Controller

The output is formulated as

$$
\begin{aligned}
Y(s)= & \frac{H(s) \cdot C(s) \cdot G(s)}{1+(C(s)+F(s)) G(s)} \times R(s) \\
& +\frac{G(s)}{1+(C(s)+F(s)) G(s)} \times D(s)
\end{aligned}
$$

The output equation above is formed by two components. The first is generated by the input $R(s)$ and the second is generated by the external disturbance $D(s)$. Now construct the algorithm in a form of expectation composition. Firstly, determine the operable elements $C(s)$ and $F(s)$ according to the second component to obtain the best resisting effect on external disturbance $D(s)$ when the disturbance changes. The control algorithm is depicted as

$$
C(s)+F(s)=K_{p}\left(1+\frac{1}{1+T_{I} S}+\frac{T_{D} S}{1+\eta T_{D} S}\right)
$$

Then determine the first operable component $H(s) \cdot C(s)$ in equation (5) by equation (7) to obtain the best tracking effect for output to input. The control algorithm is described as

$$
H(s) \cdot C(s)=K_{p}\left(\alpha+\left(\frac{1}{T_{I} S}-\frac{\beta}{1+T_{I} S}\right)+\frac{\gamma T_{D} S}{1+\eta T_{D} S}\right)
$$

For fixed set point control, the output of the target value filter should be equal to the input. According to the finite-value theorem, we have

$$
\lim _{t \rightarrow \infty} h(t)=\lim _{s \rightarrow 0} H(s)=1
$$

Then combine equation (6) and (7), we obtain the expression of target value filter $H(s)$ as

$$
H(s)=\frac{1+\alpha T_{I} S}{1+T_{I} s}+\frac{T_{I} s}{1+T_{I} s} \times\left(\frac{-\beta}{1+T_{I} s}+\frac{\gamma T_{D} s}{1+\eta T_{D} S}\right)
$$

Denoted by $\alpha, \beta$ and $\gamma$ the TDF coefficients for proportional gain, integral time constant and derivative time constant (generally $0 \leq \alpha \leq 1,0 \leq \beta \leq 1,0 \leq \gamma \leq 2$ ). And $1 / \eta$ is the differential gain (generally $0.1 \leq \eta \leq 1$ ).

Equation (9) satisfies the limit in equation (8). Then the detailed structure of our designed target value filter type TDF-PID controller is depicted in Figure 3.

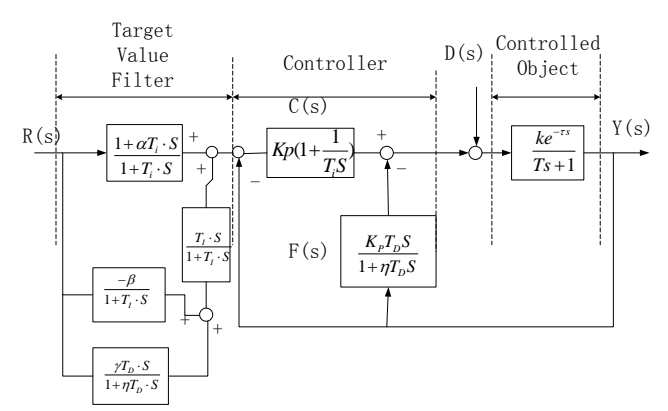

Figure 3: Structure of Target Value Filter TDF-PID Controller

\section{SIMULATION AND RESEARCH}

TDF control is to decompose the controller $C(s)$ into two parts by several design methods, one for resisting external disturbance and the other one for objective tracking. These two parameters can be adjusted separately and reach the best simultaneously. We choose the controlled object as $G(s)=\frac{3}{5 s+1} \cdot e^{-1.5 s}$, add our target value filter $H(s)$ to the one-degree of freedom PID controller, and then set the PID parameters by Z-N (Ziegler Nichols) method. The parameter values are listed in Table 1 . The performance comparison of ODF-PID and TDF-PID control systems is depicted in Figure 4.

Table 1: TDF-PID Parameters

\begin{tabular}{|ccccc|}
\hline Type & Integrated Control Method & $\alpha$ & $\beta$ & $\gamma$ \\
\hline I & P-PID (P as TDF) & 0.4 & 0 & 0 \\
II & PI-PID (PI as TDF) & 0.4 & 0.15 & 0 \\
III & PID-PID (PID as TDF) & 0.4 & 0.15 & 0.48 \\
\hline
\end{tabular}

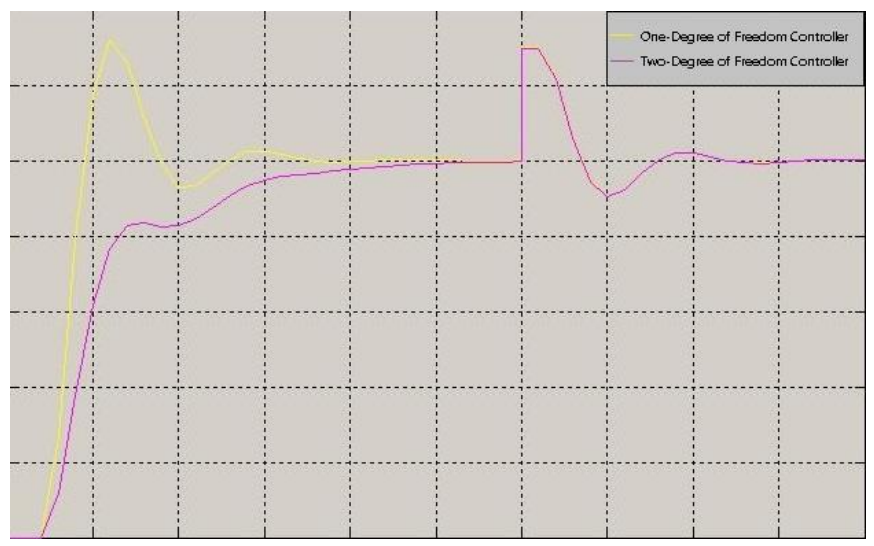

Figure 4: Performance Comparison of ODF-PID \& TDF-PID

The pink and yellow curves in Figure 4 describe the dynamic responses of TDF-PID and ODF-PID respectively. We can see the tracing performance of TDF-PID has been improved and the overshoot has been greatly decreased. So we conclude that TDF-PID can satisfy the best performance in both resisting external disturbance and tracking.

\section{ANALYSIS AND CONCLUSION}

For the typical controlled object electric oven, the static gain $k$, inertia time constant $T$ and lag time constant $\tau$ varies when temperature changes. Also, the model establishment is imprecise, so we mainly discuss the parameter uncertainty of the controlled object $G(s)=\frac{3}{5 s+1} \cdot e^{-1.5 s}$ in system robustness analysis.

Establish the accurate model with inertia time constant $T$ and static gain $k$ unchanged. Change the lag time constant $\tau$ to $1.5 \mathrm{~s}, 2.0 \mathrm{~s}, 2.9 \mathrm{~s}$ and obtain the response curves in Figure 5, colored as pink, blue and yellow respectively. When the lag time constant is greater than 2.8s, the response curve will diverge, so the upper limit of the TDF-PID system delay function is $+97 \%$. 


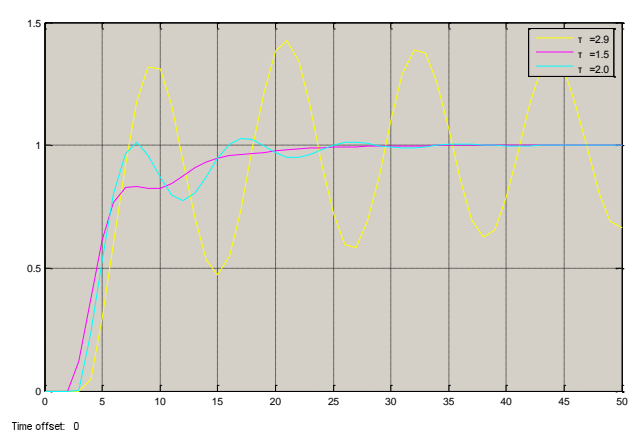

Figure 5: Lag Time Constant Changing Effect

Establish the accurate model with lag time constant $\tau$ and static gain $k$ unchanged. Change the inertia time constant $T$ to 1.6, 30,50 and obtain the response curves in Figure 6, colored as red, blue and yellow respectively. When the inertia time constant $T$ rises, the overshoot of the system output increases, the oscillation amplitude of the response enlarges, and the control force of the controller weakens, but the system can still remain stable with the oscillation frequency going down. However, when $T$ decreases, the oscillation frequency increases and tends to be diverging, and the system becomes unstable. With $T$ smaller than 1.6, the controller cannot keep the system stable. Establish the accurate model with lag time constant $\tau$ and inertia time constant $T$ unchanged. Change $k$ from 3 to 1,5 , 5.88 and obtain the response curves in Figure 7. According to the response curves, when $k$ increases, the system response oscillates more intensively and tends to be unstable. The control force of the controller reaches the limit when $k=5.88$, and the system will diverge if $k$ is greater than 5.88 . When $k$ decreases, the overshoot reduces, the rapidity becomes worse, and the system stability becomes better.

Based on the simulation results above, our designed TDF-PID controller can well solve the problem of temperature control for electric oven. This scheme not only has good performance in tracking feature and robustness with a simple structure, but also has extensive application prosperity.

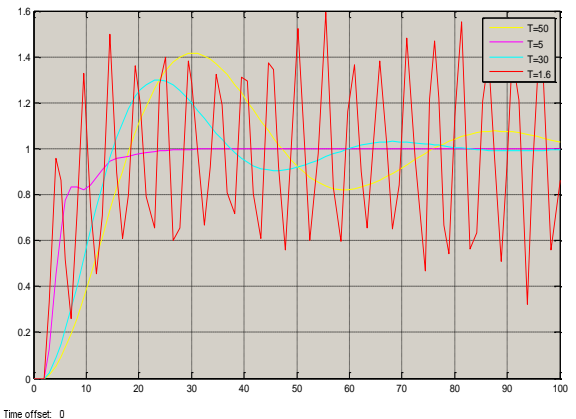

Figure 6: Inertia Time Constant Changing Effect

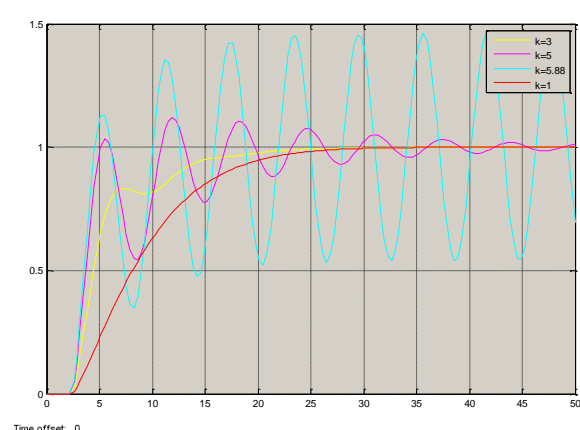

Figure 7: Static Gain Changing Effect

\section{REFERENCES}

[1] Guojuan, L., Gang, W., Meisheng, X., Dazhi, Y. 2003. Oven temperature control system based on stair-like dynamic matrix control [J]. Journal of Southeast University (Natural Science Edition), S1, 150-153.

[2] Guangping, L. 2013. Design and realization of central air-conditioning monitoring training system [J]. Automation \& Instrumentation, 05, 166$168+170$.

[3] Jinggang, Z., Run, P., Zhiyuan, L. 2002. Robust Two-degree-of-freedom PID Control [J]. Chinese Journal of Scientific Instrument, S2, 819$820+824$.

[4] Shuqiu, G., Qingding, G., Xiying, D., Haiyan, R. 2007. Two degree of freedom PID control for PMSM [J]. Journal of Shenyang University of Technology, 05, 542-545.

[5] Chongzhi, F., Deyun, X. 1988. Process Identification [M]. Beijing: Tsinghua University Press.

\section{ABOUT THE AUTHORS}

Sun Mingge (1971-): Male, Yushu City, Jilin Province, received doctor degree from Jilin University in 2010, professor of Jilin Institute of Chemical Technology, mainly researching on industrial automatic control and machine vision.

Zhao Junming (1995-): Male, Jilin City, Jilin Province, postgraduate of Jilin Institute of Chemical Technology, mainly researching on industrial automatic control.

Ren Juan (1989-): Female, Jilin City, Jilin Province, received master degree from Northeast Electric Power University in 2016, engineer in the mold department of Jilin Koito Dongguang Automobile Lamp Co., Ltd.

Li Jiayi (1986-): Female, received master degree from Jilin University in 2011, lecturer of Jilin Institute of Chemical Technology, mainly researching on control engineering, control theory and control system simulation.

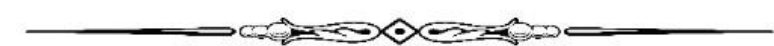

